\title{
Effect of pessary cleaning and optimal time interval for follow-up: a prospective cohort study
}

\author{
Susanne D. Thys ${ }^{1} \cdot$ Robert A. Hakvoort $^{2} \cdot$ Joyce Asseler $^{3} \cdot$ Alfredo L. Milani $^{4} \cdot$ Astrid Vollebregt $^{3} \cdot$ Jan Paul Roovers $^{1}$
}

Received: 30 August 2019 / Accepted: 25 November 2019 / Published online: 6 January 2020

(C) The Author(s) 2020

\begin{abstract}
Introduction and hypothesis The objective of this study was to determine the efficacy of routine follow-up visits for pessary cleaning, the effect of extended time intervals between visits and the proportion of patients being able to self-manage their pessary for pelvic organ prolapse (POP).

Methods We conducted a prospective cohort study in patients with a stage $\geq$ II POP without previous POP surgery. All patients received a pessary as primary treatment. Our main outcome measure was a difference $\geq 2$ in median visual analogue scale (VAS) scores (for pain, discharge, irritation) 1 week before and 1 week after cleaning. Measurements were performed after 3- and 9month cleaning intervals. For the evaluation of the effect of cleaning, 132 patients ( 3 months' follow-up) and 87 patients (12 months' follow-up) were available for analysis. For the evaluation of the effect of the lengthening interval, 123 patients were available.

Results Self-management was performed in $45.2 \%$ of patients at 1 year. In $93.1 \%$ of patients, no differences were observed in pre-and post-cleaning VAS scores (effect of cleaning) on vaginal pain. Nor was there a difference in discharge (72.4\%) or irritation $85.1 \%(p=0.00)$. No differences were observed in pre-cleaning VAS scores for vaginal pain, discharge and irritation when the interval was lengthened from 3 to 9 months. No serious adverse events occurred.

Conclusions There is no proven benefit of regular follow-up visits to clean a pessary. Also, the length of the cleaning interval does not seem to matter.
\end{abstract}

Keywords Pessary $\cdot$ Cleaning $\cdot$ Follow-up interval $\cdot$ Self-management $\cdot$ Pelvic organ prolapse

$\begin{array}{ll}\text { Abbreviations } \\ \text { POP } & \text { Pelvic organ prolapse } \\ \text { VAS } & \text { Visual analogue scale } \\ \text { POP-Q } & \text { Pelvic organ prolapse quantification } \\ \text { IRB } & \text { Institutional review board } \\ \text { SUI } & \text { Stress urinary incontinence } \\ \text { CRF } & \text { Case record form } \\ \text { SPSS } & \text { Statistical Package for the Social Sciences }\end{array}$

Susanne D. Thys

SD.Thys@gmail.com

1 Amsterdam University Medical Center, Meibergdreef 9, 1105, AZ Amsterdam, The Netherlands

2 Martini Hospital, Groningen, The Netherlands

3 Spaarne Gasthuis, Hoofddorp, The Netherlands

4 Reinier de Graaf Groep, Delft, The Netherlands

\section{Introduction}

Pelvic organ prolapse (POP) is a common condition in adult women $[1,2]$. The prevalence of symptomatic POP in the general population is $11.4 \%$ (4-12.2\%) [2]. Apart from pelvic floor muscle training, there are two other management options for POP; the use of a vaginal pessary and pelvic reconstructive surgery. As a non-surgical treatment option, vaginal pessaries are often used as first-line treatment in patients who do not want surgery, patients who are at a higher risk of surgical complications or in patients with recurrent POP [1]. Reasons to start pessary therapy are reducing POP-related symptoms, preventing progression of prolapse and avoiding surgery $[1,3]$.

Compared with surgery, advantages of pessary use are the low rate of complications and the immediate effectiveness [4-6]. However, disadvantages also exist. The most frequently described side effects of pessaries are vaginal discharge (25$33 \%$ ), vaginal irritation/pain (2-33\%) and vaginal blood loss $(6-46 \%)[4,5,7-10]$. Rarely, cases of more serious adverse 
events of pessary use are described, such as fistula, hydronephrosis, urosepsis, incarceration and carcinoma $[1,4,5$, 11]. However, it must be noted that these serious adverse events are often due to longstanding neglect of pessaries $[4,5,12]$.

Whether a pessary will be chosen as first-line treatment depends on expectations about the effect, the likelihood of occurrence of side effects and patients' preferences $[13,14]$ and existing guidelines advising to try the least invasive option initially [6]. Overall, in the Netherlands, this has resulted in high acceptance, with up to $98 \%$ of clinicians reporting use of pessaries in the management of POP and even $77 \%$ of gynaecologists reporting use of pessaries as first-line treatment $[15,16]$.

With this widespread use of pessaries, it becomes even more relevant to optimise management protocols. In comparison with surgery, short-term costs of pessaries are lower. However, long-term costs are highly dependent on the need and frequency of follow-up visits. These follow-up visits involve removal and cleaning of the pessary, inspection of the vaginal wall and afterwards replacing the pessary. These visits can be a burden to patients. Equally important, we do not know whether these visits are effective in reducing pessary related side effects. Further, intervals between these followup visits vary widely and studies about the ideal length of such intervals are lacking $[1,7,11,16,17]$. Nor is it known what proportion of patients is willing and able to learn how to clean and replace the pessary themselves.

To optimize treatment protocols and optimize the number of follow-up visits, we performed a prospective cohort study to evaluate whether or not cleaning a pessary has any effect on pessary-related symptoms such as vaginal discharge, vaginal irritation and pain. In addition, we evaluated if pessary-related side effects prior to the cleaning differ when the interval is significantly lengthened from 3 to 9 months.

\section{Materials and methods}

\section{Study design and setting}

A prospective cohort study of women with symptomatic pelvic organ prolapse (POP) and a POP-Q [18] stage 2 or higher was performed between January 2014 and December 2017 to determine the percentage of continued users after 12 months. In this cohort, several secondary outcomes were measured: disease-specific quality of life, sexual function after 1 year, factors related to discontinuation of pessary use, the effect of cleaning a pessary and pessary-related complaints when intervals between visits were increased from 3 to 9 months' follow-up. In this article we present the data concerning:

- The effectiveness of cleaning a pessary regarding the complaints of vaginal pain, discharge and irritation.
- The comparison between a 3-month cleaning interval with a 9-month interval concerning the complaints of vaginal pain, discharge and irritation.

- The study was conducted in three teaching hospitals. Study approval was obtained from the Institutional Review Board (IRB).

\section{Participants}

All patients who visited the outpatient clinic with primary, symptomatic POP (feeling or seeing a bulge) and a prolapse stage 2 or higher according to the POP-Q classification were eligible to participate and consequently given information about the study. Patients who completed the 3-month follow-up visit with the intention of continuing pessary use were included in this study. Patients with previous pelvic reconstructive surgery for POP or stress urinary incontinence (SUI) were not included.

\section{Study procedure and follow-up}

When treatment for prolapse was preferred, all patients initially received a silicone ring pessary (with or without support). Choice regarding the size of pessary was left to the discretion of the gynaecologist. All patients were offered education concerning self-management, which included practical explanation about removal, cleaning and replacement of the pessary. In the case of vaginal bleeding or excessive vaginal discharge, patients were instructed to visit their gynaecologist before the intended first follow-up visit.

The following baseline characteristics were noted in a case record form (CRF): age, parity, POP-Q stage, presence of vaginal atrophy and any prescription of local oestrogen treatment, complaints of concomitant SUI and sexual activity.

The first standard follow-up visit after insertion of the pessary was 3 months after initial placement of the pessary. In this visit, the pessary was removed and cleaned. The vaginal wall was inspected for lesions. When there were no signs of complications and the patient wished to continue pessary treatment, the pessary was replaced. To measure the effect of pessary cleaning, patients were asked to rate three known pessary-related side effects using a 1-10 visual analogue scale (VAS): vaginal pain, discharge and irritation [4, 7]. On this scale, the value 1 reflects no complaints of this side-effect and 10 reflects very severe complaints of this side-effect. This measurement was performed 1 week prior to the follow-up visit and 1 week after this follow-up visit. As a wide range of (clinically relevant) differences in VAS scores was reported and, taking errors of this measurement tool into account, we considered a difference in VAS scores of $\geq 2$ to be a clinically significant difference [19]. To answer the second question, whether or not pessary-related side effects differ when 
intervals increase from 3 to 9 months, only measurements before follow-up visits were needed.

In addition, patients received a self-developed questionnaire about whether or not they had performed self-management. If they had performed self-management, patients were asked about the frequency of self-management (daily, weekly, monthly). The second follow-up visit was 12 months after insertion of the pessary (9-month interval). Before, during and after this visit the same procedure was repeated as described above.

A sub-analysis was performed on the effect of cleaning and lengthening of the cleaning interval between patients with and without self-management.

\section{Statistical analysis}

All data were entered and analysed in a Statistical Package for the Social Sciences (SPSS Statistics for Windows, version 24.0; IBM, Armonk, NY, USA). Descriptive analyses were performed as appropriate for the respective variable. First, the normality of the variable was determined. Means with standard deviations were given for continuous, normally distributed data, medians with quartiles for not normally distributed continuous data and frequencies with percentages for categorical data. Comparisons were made using Wilcoxon signed rank test for continuous not normally distributed paired data, Mann-Whitney $U$ test for continuous not normally distributed unpaired data and Chi-squared testing for categorical data. A $p$ value of less than 0.05 was considered a threshold for statistical significance.

\section{Results}

Between January 2014 and December 2016, there were 365 patients eligible for the prospective cohort study. A total of 163 pessary users continued the use of a pessary after 3 months' follow-up and were included. Of these patients, there were 132-paired measurements before and after cleaning at 3 months' follow-up and 87 paired measurements before and after cleaning at 12 months' follow-up. With these measurements, it could be investigated whether cleaning a pessary had any effect on pessary-related complaints (i.e. vaginal pain, discharge and irritation). Of 123 patients, there was a pre-VAS score measurement available at both the 3- and the 12-month follow-up (Fig. 1).

Figure 1 shows the patients who were lost to follow-up and the missing data. In total, 5 patients (3\%) were lost to follow-up. One of them died (not pessary-related) and the other four did not return to any follow-up visit, nor did they respond to requests to make an appointment. Eleven $(6.7 \%)$ patients discontinued pessary use, of which 7 (4.3\%) underwent pelvic reconstructive surgery. Four patients refused further pessary treatment and operative treatment for POP for several reasons, e.g. discharge complaints and no wish for surgery. Further, in one patient treatment was temporarily stopped because of a lesion of the vaginal epithelium due to pessary use, one patient changed to a cube pessary and the other patient only used a pessary while exercising. All patients in the "missing data" group continued pessary treatment (according to the patient record files).

Table 1 shows the baseline characteristics of the study population (and divides into patients with and without self-management). The mean age was 65 years (75-72), of which the majority were post-menopausal $(88.9 \%)$. Median parity was 2 (range 2-3). One hundred and one $(64.3 \%)$ patients had a POP-Q classification stage 2, $55(35.0 \%)$ stage 3 and 1 $(0.7 \%)$ had stage 4 prolapse. Vaginal atrophy was present in 63 patients $(44.1 \%)$ of whom $51(81 \%)$ were treated with vaginal oestrogen. Concomitant SUI was reported in 32 patients $(21.2 \%)$. Sexual activity was present in $50.3 \%$ of our study population, $94 \%$ of them with vaginal penetration.

Patients who performed self-management were younger (median 63 vs 67 years respectively $p=0.04$ ), were more frequently diagnosed with vaginal atrophy (60 versus $28.8 \%$ respectively, $p=0.00)$, reported less concomitant SUI (80.8 versus $74.6 \%$ respectively, $p=0.01$ ) and were more often sexually active (including vaginal penetration; 61.1 vs $38.2 \%$ respectively; $p=0.03$ ).

The effect of cleaning is shown in Tables 2 and 3. Overall, no significant differences were found in pre- and postcleaning VAS scores (difference of $\geq 2$ points on VAS scores) on vaginal pain, discharge and irritation at the 3 - and 12month follow-up visits ( $p=0.00$ on all vaginal symptoms, in favour of the "no difference" groups). Nevertheless, a number of patients (up to $23 \%$ after a cleaning interval of 9 months) tended to have some improvement in vaginal discharge complaints after cleaning. The sub-analysis in patients with selfmanagement and without self-management showed that there were no differences between the two groups (Table 3).

No significant differences were observed in vaginal pain (median pre VAS scores $1, p=0.25$ ), discharge (median pre VAS scores 3, $p=0.23$ ) and irritation (median pre VAS scores $1, p=0.70$ ) when the interval of cleaning was increased from 3 months and 9 months respectively.

Self-management was performed in $45.2 \%$ of included patients compared with $42.9 \%$ who did not at 12 months' followup. A remaining $8.2 \%$ did not perform self-management at 3 months' follow-up, but no information about these patients was available at 12 months' follow-up. In $3.7 \%$ of patients, there was no information about self-management at all. Frequencies of performing self-management showed that $5.4 \%$ of patients performed this daily, $31.1 \%$ weekly, $40.5 \%$ monthly and $6.8 \%$ yearly. Another $16.2 \%$ performed selfmanagement only if they thought it was necessary, of which 8 $(10.2 \%)$ occasionally went to the general practitioner for help.

No serious adverse events due to pessary use were recorded in our study group after 12 months' follow-up. 
Fig. 1 Flowchart.

*Measurements in the same

patient. All patients with missing

data have continued pessary use

at 12 months' follow-up
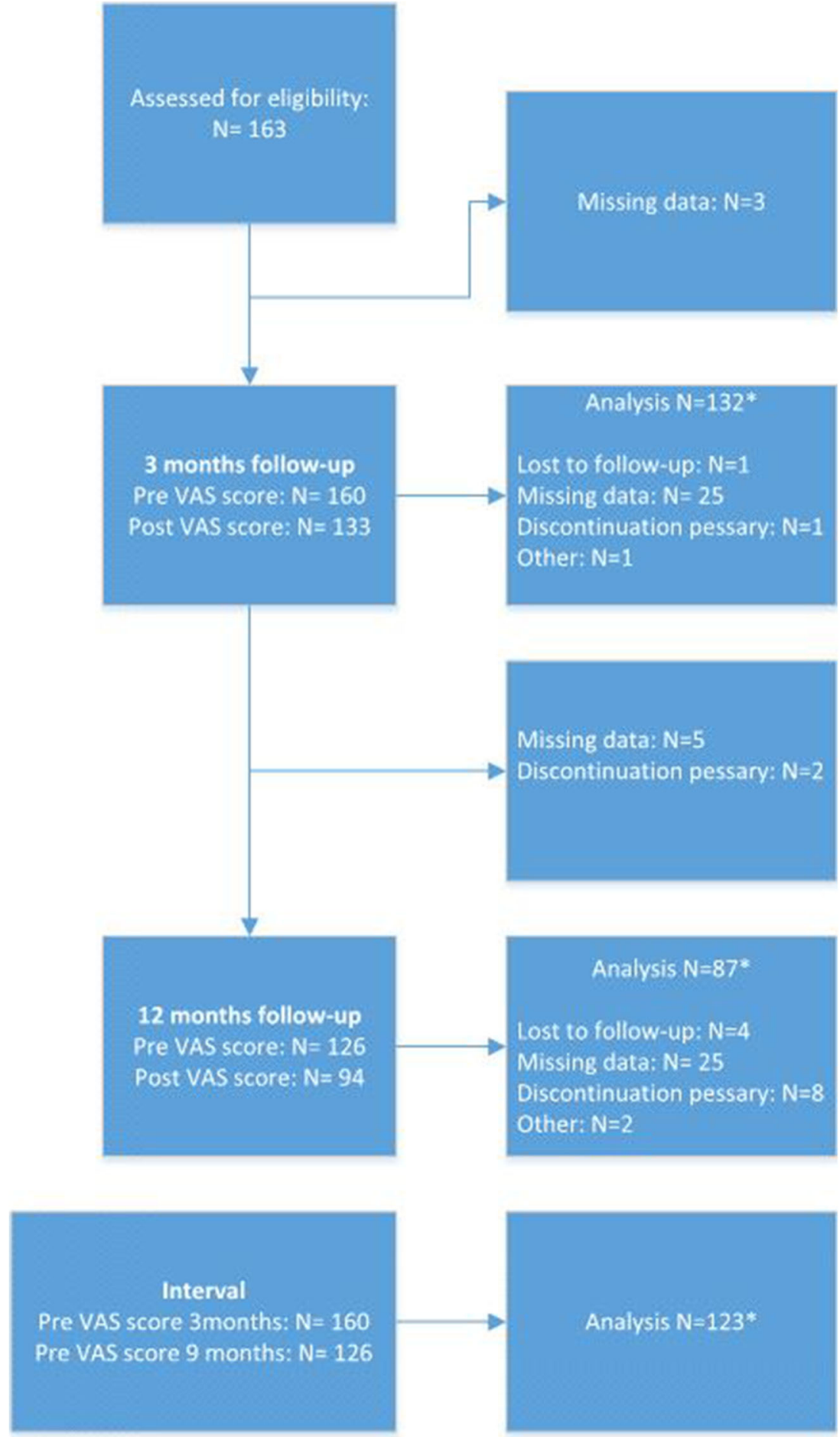

\section{Discussion}

\section{Main findings}

Cleaning a pessary, either by a physician or by the patient herself, does not improve pessary-related side effects. An interval of 6 months between cleaning visits did not result in an increase in pessary-related side effects.

\section{Strengths and limitations}

Strengths of this study are the prospective design (with preand post-cleaning measurements) and the relatively large group of patients.

Another strength is that in this study we take a closer look at pessary management and routine follow-up visits. Although there is a wide range of opinions 
Table 1 Characteristics in the total study population and in women with or without self-management

\begin{tabular}{|c|c|c|c|c|c|c|c|}
\hline \multirow[b]{2}{*}{ Age } & \multicolumn{2}{|c|}{ Total $(N=163)$} & \multicolumn{2}{|c|}{ No self-management $(N=70)$} & \multicolumn{2}{|c|}{ Self-management $(N=74)$} & \multirow{2}{*}{$\frac{p \text { value }}{0.04 *}$} \\
\hline & 65 & $57-72$ & 67 & $60-74$ & 63 & $56-70$ & \\
\hline Parity & 2 & $2-3$ & 2 & $2-3$ & 2 & $2-3$ & $0.52 *$ \\
\hline \multicolumn{8}{|l|}{ Menopausal state } \\
\hline Premenopausal & 11 & 6.8 & 2 & 3.0 & 7 & 9.7 & \multirow[t]{3}{*}{$0.39 * *$} \\
\hline Perimenopausal & 7 & 4.3 & 4 & 6,00 & 3 & 4.2 & \\
\hline Postmenopausal & 143 & 88.9 & 61 & 91.0 & 62 & 86.1 & \\
\hline \multicolumn{8}{|l|}{ POP-Q } \\
\hline Stage 2 & 101 & 64.3 & 47 & 72.3 & 40 & 54.8 & \multirow[t]{3}{*}{$0.08^{* *}$} \\
\hline Stage 3 & 55 & 35.0 & 18 & 27.7 & 32 & 43.8 & \\
\hline Stage 4 & 1 & 0.7 & 0 & 0 & 1 & 1.4 & \\
\hline \multicolumn{8}{|l|}{ Vaginal atrophy } \\
\hline Yes & 63 & 44.1 & 17 & 28.8 & 42 & 60.0 & \multirow[t]{3}{*}{$0.00 * *$} \\
\hline Vaginal HT & 51 & 35.7 & 12 & 20.3 & 37 & 52.9 & \\
\hline No & 80 & 55.9 & 42 & 71.2 & 28 & 40.0 & \\
\hline \multicolumn{8}{|l|}{ SUI } \\
\hline Yes & 32 & 21.1 & 15 & 25.4 & 14 & 19.2 & \multirow[t]{2}{*}{$0.01 * *$} \\
\hline No & 119 & 78.8 & 44 & 74.6 & 59 & 80.8 & \\
\hline \multicolumn{8}{|l|}{ Sexually active } \\
\hline Yes & 80 & 50.3 & 26 & 38.2 & 44 & 61.1 & \multirow[t]{3}{*}{$0.03 * *$} \\
\hline Penetration & 75 & 47.2 & 25 & 36.8 & 41 & 56.9 & \\
\hline No & 79 & 49.7 & 42 & 61.8 & 28 & 38.9 & \\
\hline
\end{tabular}

Data are median (quartiles) or numbers (percentage)

SUI stress urinary incontinence

*Mann-Whitney $U$ test, **Chi-squared test

regarding (optimal) pessary management, up to now evidence is still scarce.

Some limitations of our study should be considered.

First, we have to acknowledge that our overall population was a subgroup of successful pessary users, without complications, e.g. lesions of the vaginal epithelium or serious adverse events. Although this could have resulted in a bias, the main study question was to determine the efficacy of cleaning; this inherently applies to patients who are in fact successful users.

Second, comparison of the 3 months and 9 months cleaning intervals might be deceptive. It is possible that the measurement of vaginal symptoms after a 9-month interval is actually a reflection of a bacterial environment that had already colonized the vagina in the preceding 6 months. What we can conclude is that lengthening the cleaning interval does not increase discharge and therefore most probably does not lead to an increase in colonization [8-10].

Third, the inclusion of patients performing selfmanagement may have influenced cleaning and interval results in this group of patients. However, we decided to offer education on self-management to all of our patients, as the ability for self-management is desirable; the self-reliance of patients increases and the need for a doctor's visit diminishes. As a result, instruction for self-management is increasingly becoming part of normal routine. Therefore, we decided to include this group [20, 21].

Finally, there was a considerable amount of missing data in this study. Most of the missing data consisted of post-visit
Table 2 Pre- and post-cleaning VAS scores on vaginal symptoms

\begin{tabular}{|c|c|c|c|c|c|c|c|c|c|c|}
\hline \multirow[b]{3}{*}{ Vaginal pain } & \multicolumn{5}{|c|}{3 months $(N=132)$} & \multicolumn{5}{|c|}{12 months $(N=87)$} \\
\hline & \multicolumn{2}{|c|}{ Pre-VAS } & \multicolumn{2}{|c|}{ Post-VAS } & \multirow{2}{*}{$\frac{p \text { value* }}{0.52}$} & \multicolumn{2}{|c|}{ Pre-VAS } & \multicolumn{2}{|c|}{ Post-VAS } & \multirow{2}{*}{$\frac{p \text { value } *}{0.67}$} \\
\hline & 1.00 & $(1-1)$ & 1.00 & $(1-1)$ & & 1.00 & $(1-1)$ & 1.00 & $(1-1)$ & \\
\hline Vaginal discharge & 3.00 & $(1-5)$ & 2.00 & $(1-3)$ & 0.17 & 3.00 & $(1-5)$ & 3.00 & $(1-4)$ & 0.00 \\
\hline Vaginal irritation & 1.00 & $(1-2)$ & 1.00 & $(1-2)$ & 0.07 & 1.00 & $(1-2)$ & 1.00 & $(1-2)$ & 0.00 \\
\hline
\end{tabular}

Scores are median (25-75 quartiles)

*Wilcoxon signed rank test 


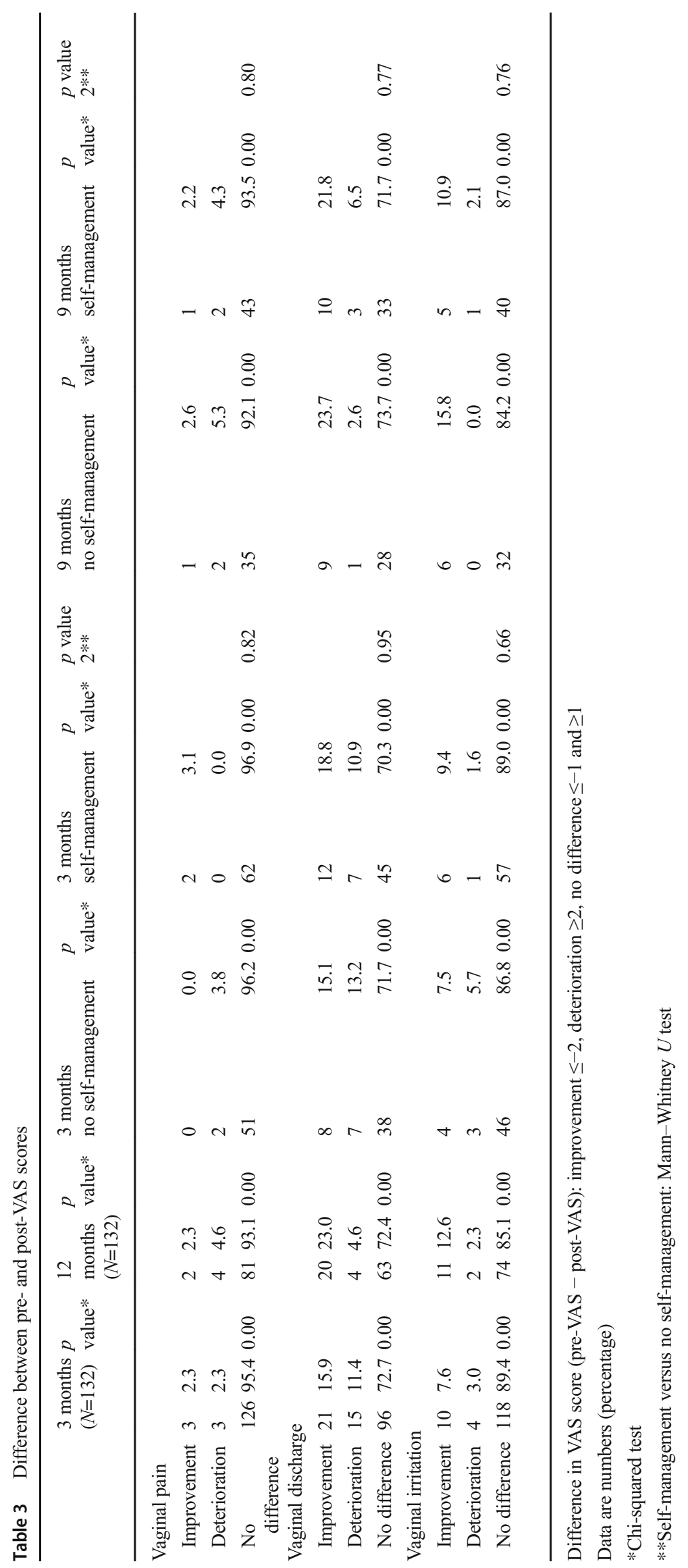


VAS measurements. Patients received the post-visit questionnaire and instructions at the respective follow-up visit. To simplify returning these documents, patients also received an addressed envelope. If the questionnaire was not returned after 2 weeks, it was noted as missing. At this time, new questionnaires were not sent because the results, when obtained too long after the specific visit, could no longer be related to the actual event of cleaning.

\section{Interpretation of results}

The first question of this study was whether the actual cleaning of a pessary is effective in reducing symptoms. In this study, no significant differences in pain, discharge and irritation were found when the situation before and after cleaning was compared.

For this finding, there can be several reasons.

First, the highest median VAS scores in pessary users were found for vaginal discharge. This is consistent with other studies on this topic $[4,5,7,8,10]$. Still, in this study we only found a median VAS score of as low as 3 on a ten-point scale (range 1-5) for vaginal discharge. A relatively low preexisting VAS score before cleaning can be partly responsible for the absence of a measurable effect of cleaning.

Second, the susceptibility for the development of vaginal discharge differs between individuals. Differences in vaginal environment and pre-existing acidity (presence of lactobacillus and atrophy) are known to influence the development of colonization and inflammation [8-10, 22].

Considering that the vagina itself is not cleaned during the cleaning visits, our hypothesis is that it is not likely that the shortterm removal of the pessary, cleaning and replacement will substantially alter bacterial colonization, inflammation or vaginal acidity. This is underlined by the fact that we did not find differences in our population between the pre- and post-cleaning visit.

The second question of this study was whether the length of the interval between cleaning visits influences pessary-related side effects (vaginal pain, discharge and irritation). It was shown that an interval of 3 months versus a 9-month interval did not influence the severity of pessary-related side effects. In the literature, it is often recommended to apply intervals of between 3 to 6 months $[1,12,16,17]$. However, we found no evidence to support these recommendations. The severity of side effects, particularly discharge (colonization of the vaginal microenvironment) appears to develop in the first period after insertion of a pessary and remains relatively stable over time [8-10]. Groups that studied the effect of pessaries on the microenvironment of the vagina showed that microscopic colonization changes accompanied by an increase in bothersome discharge already develops and occurs during the first 2 weeks of pessary use and reaches a steady state in the next 3 months [8]. Other studies have confirmed that changes in microenvironment occur mainly during the first period of pessary use $[9,10]$. The finding in our study that the severity of discharge does not increase over time is therefore in line with the literature.

Having established that intervals do not matter for the most bothersome complaints, it still might be worthwhile to remove the pessary owing to a possible chance of lesions of the vaginal epithelium and subsequent epithelial overgrowth or fistula. Other studies showed that serious adverse events in women with pessary treatment were very rare and mostly due to longstanding neglect $[4,5,12]$. In our study, we did not find any serious adverse events in patients with continued pessary use after 12 months.

Finally, our third question was which proportion of patients was willing and able to remove, clean and re-insert their pessary.

Almost half of patients were able to learn and continue selfmanagement after 12 months. Comparable percentages were described in a study by Kearney et al. They showed that $65 \%$ of patients were able to learn self-management and that $47 \%$ continued self-management after 6 months' follow-up [23].

To implement the outcomes of our study into clinical practice and subsequently reduce health care costs, we recommend a routine follow-up visit not more frequently than at 9-month intervals in a symptomatic patient without self-management. We have not studied longer intervals and therefore we do not know whether serious complications would occur at larger intervals. In our opinion, there is no evidence for the need to check asymptomatic patients performing self-management. Individualized care and visits should be provided on the indication of complaints of increasing vaginal discharge (exclude or treat bacterial vaginosis), blood loss or (increasing) pain in all patients.

\section{Conclusion}

Frequent pessary cleaning in asymptomatic women with POP is not efficacious with regard to pessary-related side effects such as pain, discharge and irritation. There is no difference in the severity of these pessary-related side effects between a 3and a 9-monthly follow-up interval.

Authors' contributions ST: protocol/project development, data collection, data analysis, manuscript writing/editing; RH: protocol/project development, data collection, manuscript editing; JA: data collection; AM: data collection, manuscript editing; AV: protocol/project development, data collection, manuscript editing; JPR: protocol/project development, manuscript editing.

\section{Compliance with ethical standards}

Conflicts of interest None.

Open Access This article is licensed under a Creative Commons Attribution 4.0 International License, which permits use, sharing, adaptation, distribution and reproduction in any medium or format, as long as you give appropriate credit to the original author(s) and the source, 
provide a link to the Creative Commons licence, and indicate if changes were made. The images or other third party material in this article are included in the article's Creative Commons licence, unless indicated otherwise in a credit line to the material. If material is not included in the article's Creative Commons licence and your intended use is not permitted by statutory regulation or exceeds the permitted use, you will need to obtain permission directly from the copyright holder. To view a copy of this licence, visit http://creativecommons.org/licenses/by/4.0/.

\section{References}

1. Bugge C, Adams EJ, Gopinath D, Reid F. Pessaries (mechanical devices) for pelvic organ prolapse in women. Cochrane Database Syst Rev 2013;2:CD004010.

2. Slieker-ten Hove MC, Pool-Goudzwaard AL, Eijkemans MJC, Steegers-Theunissen RPM, Burger CW, Vierhout ME. Symptomatic pelvic organ prolapse and possible risk factors in a general population. Am J Obstet Gynecol. 2009;200:184.e1184.e7.

3. Oliver R, Thakar R, Sultan AH. The history and usage of the vaginal pessary: a review. Eur J Obstet Gynecol Reprod Biol. 2011;156:125-30.

4. Sarma S, Ying T, Moore KH. Long-term vaginal ring pessary use: discontinuation rates and adverse events. BJOG. 2009;116:1715-21.

5. Abdulaziz M, Stothers L, Lazare D, Macnab A. An integrative review and severity classification of complications related to pessary use in the treatment of female pelvic organ prolapse. Can Urol Assoc J. 2015;9(5-6):E400-6.

6. Dutch Association Obstetrics and Gynecology, guidelines: pessary treatment for pelvic organ prolapse 2014; URL https:// richtlijnendatabase.nl/prolaps/pessarium.

7. Velzel J, Roovers JP, Van der Vaart CH, Broekman B, Vollebregt A, Hakvoort R. A nationwide survey concerning practices in pessary use for pelvic organ prolapse in the Netherlands: identifying needs for further research. Int Urogynecol J. 2015;26:1453-8.

8. Collins S, Beigi R, Mellen C, O'Sullivan D, Tulikangas P. The effect of pessaries on the vaginal microenvironment. Am J Obstet Gynecol. 2015;212:60.e1-6.

9. Meriwether KV, Rogers RG, Craig E, Peterson SD, Gutman RE, Iglesia CB. The effect of hydroxyquinoline-based gel on pessaryassociated bacterial vaginosis: a multicenter randomized controlled trial. Am J Obstet Gynecol. 2015;213:729.e1-9.

10. Fregosi NJ, Hobson DTG, Cl K, Gaskins JT, Stewart JR, Meriwether KV. Changes in the vaginal microenvironment as related to frequency of pessary removal. Female Pelvic Med Reconstr Surg. 2018;24:166-71.

11. Jelovsek JE, Maher C, Barber MD. Pelvic organ prolapse. Lancet. 2007;369:1027-38.

12. Khaja A, Freeman RM. How often should shelf/Gellhorn pessaries be changed? A survey of IUGA urogynaecologists. Int Urogynecol J. 2014;25:941-6.

13. Basu M, Wise B, Duckett J. A qualitative study of women's preferences for treatment of pelvic floor disorders. BCOG. 2011;118:338-44.

14. Thys SD, Roovers JP, Geomini PM, Bongers MY. Do patients prefer a pessary or surgery as primary treatment for pelvic organ prolapse. Gynecol Obstet Investig. 2012;74:6-12.

15. Cundiff GW, Amundsen CL, Bent AE, Coates KW, Schaffer JI, Strohbehn K, et al. The PESSRI study: symptom relief outcomes of a randomized crossover trial of the ring and Gellhorn pessaries. Am J Obstet Gynecol. 2007;196:405.e1-8.

16. Gorti M, Hudelist G, Simons A. Evaluation of vaginal pessary management: a UK-based survey. J Obstet Gynecol. 2009;29(2): 129-31.

17. Hooper GL, Atnip S, O’Dell K. Optimal pessary care: a modified Delphi consensus study. Midwifery Womens Health. 2017;62:45261.

18. Bump RC, Mattiasson A, Bo K, Brubaker LP, DeLancey JO, Klarskov $\mathrm{P}$, et al. The standardization of terminology of female pelvic organ prolapse and pelvic floor dysfunction. Am J Obstet Gynecol. 1996;175:10-7.

19. Kersten P, White PJ, Tennant A. Is the pain visual analogue scale linear and responsive to change? An exploration using Rasch analysis. PLoS One. 2014;9(6):e99485.

20. Kolle D, Kunczicky V, Uhl-Steidl M, Pontasch H. Safety and acceptance of self application of cubic pessaries and urethral ring pessaries. Gynakol Geburtshilfliche Rundsch. 1998;38:242-6.

21. Meriwether KV, Komesu YM, Craig E, Qualls C, Davis H, Rogers RG. Sexual function and pessary management among women using a pessary for pelvic floor disorders. J Sex Med. 2015;12:2339-49.

22. Yoshimura K, Morotomi N, Fukuda K, Hachisuga T, Taniguchi H. Effects of pelvic organ prolapse ring pessary therapy on intravaginal microbial flora. Int Urogynecol J. 2016;27:219-27.

23. Kearney R, Brown C. Self-management of vaginal pessaries for pelvic organ prolapse. BMJ Qual Improv Rep2014; u206180w2533.

Publisher's note Springer Nature remains neutral with regard to jurisdictional claims in published maps and institutional affiliations. 